\title{
ÉCONOMIE Économie rurale
}

RURALE

Agricultures, alimentations, territoires

368 | Avril-juin 2019

Varia

\section{Pourquoi rester en " ville moyenne »? Le cas d'entreprises agroalimentaires d'Occitanie}

Why remain located in small cities? The case of agrifood firms in Occitanie (France)

\section{Geoffroy Labrouche et Rachel Levy}

\section{(2) OpenEdition}

\section{Journals}

Édition électronique

URL : http://journals.openedition.org/economierurale/6706

DOI : 10.4000/economierurale.6706

ISSN : 2105-2581

Éditeur

Société Française d'Économie Rurale (SFER)

Édition imprimée

Date de publication : 30 juin 2019

Pagination : $35-54$

ISSN : 0013-0559

Référence électronique

Geoffroy Labrouche et Rachel Levy, «Pourquoi rester en «ville moyenne » ? Le cas d'entreprises agroalimentaires d'Occitanie », Économie rurale [En ligne], 368 | Avril-juin 2019, mis en ligne le 01

janvier 2021, consulté le 07 janvier 2021. URL : http://journals.openedition.org/economierurale/6706 ; DOI : https://doi.org/10.4000/economierurale.6706

(c) Tous droits réservés 


\title{
Pourquoi rester en « ville moyenne ॥ ? Le cas d'entreprises agroalimentaires d'Occitanie
}

\author{
Geoffroy LABROUCHE • LEREPS Toulouse; Université Toulouse II Jean Jaurès \\ geoffroy.labrouche@univ-tlse2.fr \\ Rachel LEVY • LEREPS Toulouse; ENSFEA \\ rachel.levy@ut-capitole.fr
}

Dans cet article, les auteurs proposent d'étudier les avantages et les inconvénients qui poussent les entreprises du secteur agroalimentaire à rester localisées dans des villes moyennes. Après avoir passé en revue la littérature sur les déterminants de la localisation d'entreprises dans les métropoles et les zones rurales, ils soutiennent que la ville moyenne combine les avantages de la métropole et des zones rurales. Sur la base d'entretiens semi-directifs, les auteurs étudient plus particulièrement le cas de 10 entreprises agroalimentaires localisées dans le Sud-Ouest de la France. Cette étude exploratoire permet de confirmer la spécificité des villes moyennes et de souligner le rôle crucial des politiques de soutien aux entreprises locales comme facteurs de différenciation entre villes moyennes.

MOTS-CLÉS : ville moyenne, industrie agroalimentaire, métropole, ruralité, étude de cas

\begin{abstract}
Why remain located in small cities? The case of agrifood firms in Occitanie (France)
This article examines the advantages and disadvantages of agrifood firms remaining based in small cities. After reviewing the literature on the determinants of firms' location in large cities and rural areas, we argue that small cities combine the advantages of large cities and rural areas. On the basis of semi-structured interviews, we study the case of ten agrifood firms located in the southwest of France. This study confirms the specificity of small cities and highlights the crucial role of policies aimed at supporting local businesses as a differentiating factor for small cities. (JEL: R1, Q13)
\end{abstract}

KEYWORDS: small city, agrifood industry, metropolis, rurality, case study

U En France, le déclin des villes de province est celui d'un marqueur de son identité », titrait le New York Times dans un article du 28 février 2017 à propos d'Albi, petite ville du Sud-Ouest de la France. La question du déclin des villes moyennes couplé à l'essor de la métropolisation est un sujet régulièrement abordé par les journalistes, mais aussi par les chercheurs en sciences humaines (Davezies, 2010). Les politiques territoriales impulsées ces vingt dernières années ont d'ailleurs suivi cette direction, en considérant que l'espace métropolitain devait jouer le rôle de fournisseur exclusif des ressources nécessaires au développement (Campagnac-Ascher, 2015 ; Demazière, 2017). Au niveau international, ceci se traduit par l'idée que la compétitivité des pays industrialisés repose sur des villes toujours plus denses et de moins en moins dans la périphérie. Cette thèse est cependant critiquée en France par Bouba-Olga et Grossetti (2015) et l'objet de recherche «ville moyenne» constitue une tradition d'étude qui implique différentes disciplines des sciences sociales et particulièrement la géographie (Demazière, 2014). Dans cette lignée, cet article se consacre à l'étude des villes moyennes en tant que zones urbaines spécifiques situées dans un espace intermédiaire entre les métropoles et les espaces ruraux, qui leur confère un intérêt.

Les villes moyennes ont cette spécificité d'être l'objet d'un discours depuis une 
cinquantaine d'années, porteur de valeurs vertueuses et qualitatives (Demazière, 2017). Ce discours met en avant des valeurs de convivialité villageoise et d'accès à la nature associées à une qualité de l'offre de service et d'aménagement destinée à attirer de futurs habitants ainsi que des touristes (Mainet, 2011). Cet article tente de dépasser ce discours en étudiant les spécificités en termes d'avantages et d'inconvénients que peut représenter une localisation dans une ville moyenne pour une entreprise. Il traite en particulier du cas de l'industrie agroalimentaire, qui entretient des liens étroits de proximité avec les ressources agricoles locales (Fearne et al., 2013). L'objectif de cet article est donc de faire émerger des facteurs de différenciation - du point de vue des entreprises - des villes moyennes visà-vis des territoires tant ruraux qu'urbains. Il cherche également à savoir s'il existe des facteurs de différenciation entre villes moyennes.

L'article est structuré en trois parties. La première passe en revue la littérature consacrée aux spécificités des villes moyennes mais aussi des zones métropolitaines et rurales. La deuxième présente les données et les aspects méthodologiques. Enfin, la troisième partie fait émerger les avantages et les inconvénients de la localisation en ville moyenne. Deux cas de villes présentant des caractéristiques particulières sont développés plus en détail : Cahors et Rodez.

\section{Villes moyennes, métropoles et zones rurales Principales caractéristiques et éléments de différenciation}

L'objectif de cet article est d'identifier les caractéristiques idiosyncrasiques des villes moyennes qui permettent de différencier ces espaces spécifiques des espaces urbains métropolitains d'une part et ruraux d'autre part. Afin d'atteindre cet objectif, cette première partie revient sur les principales caractéristiques et éléments de différenciation de ces trois types d'espaces que sont la ville moyenne, la métropole et la ruralité.

\section{La ville moyenne: une forme spécifique de territoire}

La ville moyenne est souvent définie comme «un objet réel non identifié » (Brunet, 1997). Elle rassemble une multitude de définitions qui tentent d'en dessiner les contours. Cependant, les auteurs s'accordent sur le fait que la ville moyenne fait référence à des aspects de taille et de fonctionnalité de la ville (Santamaria, 2000 ; Nadou, 2010), malgré des différences de points de vue tant sur les seuils de population, allant de 20000 à 30000 habitants jusqu'à 100000 à 200000 habitants, que sur l'objet d'étude analysé : ville centre ou aire urbaine. Levy et al. (2013) ont défini les villes moyennes comme des aires urbaines de 30000 à 200000 habitants (de la même manière que les travaux menés à l'échelle européenne dans le cadre de l'ESPON (2008), alors que Floch et Morel (2001) les définissent comme des villes de 20000 à 100000 habitants. Les villes moyennes sont également définies comme des zones «non métropolitaines » reconnues pour leur rôle organisationnel sur le territoire: administration diversifiée, système éducatif développé, structures de santé, services aux individus et aux entreprises, vie intellectuelle et parfois structures universitaires (Santamaria, 2000). Elles ont un rôle important à jouer dans l'évolution des modes de vie et en particulier dans la satisfaction du désir de nature des individus. Les évolutions à la hausse des prix du foncier et de l'immobilier métropolitains, l'image négative de la grande ville, la concentration de la vie sociale sur la sphère familiale et l'accroissement des mobilités tendent à renforcer le rôle de ces villes de taille inférieure (Bailly et Bourdeau-Lepage, 2011). Certaines études remettent en cause 
l'effet des métropoles sur le développement économique au profit des villes de « second rang ». En particulier, Camagni et al. (2015) montrent que les villes de taille plus modeste compensent les plus faibles effets d'agglomération en développant un certain nombre de fonctions et notamment celle de connexion avec d'autres villes.

La ville moyenne renvoie donc à une notion de relais, un lieu d'intermédiation dans l'armature urbaine d'un pays ou d'une région (Charbonneau et al., 2003 ; Zuliani, 2004 ; Nadou, 2010). Elle conserve une primauté forte sur la fourniture de services aux entreprises et à la population, exerçant ainsi une fonction « quasi métropolitaine » sur leur hinterland (Zuliani, 2004). Ce rôle structurant de l'espace entre la métropole et le rural à travers des fonctions socioéconomiques importantes met en exergue une nécessité de double connectivité (Nadou, 2010). En effet, la ville moyenne se caractérise par une connectivité interne vis-à-vis de son hinterland via des fonctions utilitaires. Mais elle bénéficie également d'une connectivité externe vers le niveau métropolitain, voire national. $\mathrm{La}$ connectivité de la ville moyenne permet ainsi de raccrocher des acteurs ruraux à des réseaux de services, d'emploi ou d'équipement qui seraient difficiles à atteindre sans cet intermédiaire.

Il convient, avant de se risquer à caractériser plus en détail les villes moyennes, de les distinguer du «périurbain » ou de la « rurbanisation ». Le concept de périurbain exprime la diffusion de l'urbain dans le rural en lien avec l'évolution des modes de vie (Huriot et Bourdeau-Lepage, 2009). Ces espaces situés autour de la ville se caractérisent par un cadre de vie plutôt rural avec des habitants qui travaillent à la métropole à travers des déplacements quotidiens. Ces espaces, principalement résidentiels, sont donc polarisés et caractérisés par de l'étalement urbain et des zones pavillonnaires (Mora et al., 2012).
On parle de «ruralité résidentielle » (Huriot et Bourdeau-Lepage, 2009 ; Bailly et Bourdeau-Lepage, 2011). Le périurbain fait donc partie de la ville (Bailly et Bourdeau-Lepage, 2011), la ville moyenne pouvant d'ailleurs elle-même disposer d'espaces périurbains.

La ville moyenne constitue donc un espace urbain spécifique situé en position intermédiaire entre la métropole et la campagne rurale. Cet espace peut ainsi combiner les avantages des zones métropolitaines et rurales, en particulier pour les entreprises agroalimentaires qui ont besoin de se trouver à proximité des ressources agricoles ainsi que d'accéder à un certain nombre de services. De ce constat émerge une première hypothèse :

H1: Les entreprises agroalimentaires localisées dans des villes moyennes tirent parti de manière conjointe des avantages urbains et ruraux sans en subir les inconvénients.

Une fois cette hypothèse validée, il s'agira de détailler grâce à notre étude les avantages (urbains et ruraux) dont mettent à profit les entreprises agroalimentaires localisées dans des villes moyennes.

Les villes moyennes possèdent une matérialité fortement inscrite dans l'histoire et les cultures locales (Guay et Hamel, 2004) et la gamme des systèmes économiques qu'elles sont susceptibles de développer est étendue (Carrier et Demazière, 2012). En effet, ces systèmes dépendent en grande partie de l'appareil industriel de la ville, du développement des services à l'entreprise (Zuliani, 2004) ou encore de la présence d'universités. En conséquence, la littérature met en avant des trajectoires différentes au sein des villes moyennes. Lorsque certains auteurs soulignent un recul de l'emploi dans les villes moyennes (Davezies et Pech, 2014), d'autres observent, au contraire, un développement de ces dernières (BoubaOlga et al., 2012). C'est pourquoi une seconde hypothèse émerge : 
H2: Au sein des villes moyennes, il existe des critères de différenciation qui expliquent des différences de dynamisme économique.

En cas de validation de cette hypothèse, il s'agira de déterminer ces critères de différenciation.

Afin de mieux cerner les spécificités des villes moyennes, les caractéristiques des zones métropolitaines et rurales vont être passées en revue.

\section{La métropole : espace urbain de référence}

Les villes moyennes sont avant tout des villes, c'est-à-dire des espaces urbanisés. L'espace urbanisé par excellence demeure, semble-t-il, la métropole. En effet, l'idée de la métropole comme moteur de la croissance économique et du développement est une idée répandue dans la communauté scientifique qui influence directement les réformes politiques (Bouba-Olga et Grossetti, 2015). Les espaces métropolitains se caractérisent également par des taux d'innovation supérieurs (Malmberg, 1997) induits par la concentration géographique des acteurs.

Pour les tenants de la Nouvelle Économie Géographique, Krugman (1991) notamment, les logiques d'agglomération sont le moteur de la dynamique des échanges. Ainsi, la concentration spatiale permet de bénéficier de rendements croissants et d'une réduction des coûts de transaction laissant penser que la concentration spatiale des activités serait la clé du développement économique notamment en France (Davezies et Pech, 2014). Pour les entreprises, ces espaces se caractérisent aussi par des accès facilités aux infrastructures de transport et aux plateformes logistiques (Mayneris, 2017), à une maind'œuvre qualifiée (Reynaud et Bouvet, 2016), voire à une plus grande diversité de la main-d'œuvre qualifiée (Frenken et Boschma, 2007).
Ces espaces se caractérisent aussi par l'accès facilité aux externalités positives issues des effets d'agglomération (Billings et Johnson, 2016) qui permettent ainsi d'atteindre une masse critique d'investissements et d'infrastructures qui favoriseraient l'entrepreneuriat et l'innovation (Pezzini, 2001; Audretsch et Feldman, 2004). Ces espaces peuvent conduire à l'émergence de clusters (Porter, 1998). Ceux-ci regroupent une variété d'entreprises et d'acteurs spécialisés dans un domaine et permettent d'accroître la compétitivité économique par des dynamiques d'innovation, amplifiées par les effets d'agglomération ; même si certains auteurs (Becattini, 1992) ont pu mettre en évidence l'existence de districts industriels dans des territoires non métropolitains. Les métropoles sont également vues comme le lieu où réside la classe créative (Florida, 2002). Les effets d'agglomérations se retrouvent de la même manière dans les sphères culturelle et créative et dans l'offre culturelle (Guillon et Ambrosino, 2016).

Ces différentes caractéristiques se retrouvent dans une certaine mesure dans les villes moyennes. Tallec (2012) montre par exemple que l'innovation peut se produire en dehors des espaces métropolitains et en particulier dans les villes moyennes. Guéraut (2017) montre, quant à lui, que les villes moyennes connaissent un retour de main-d'œuvre qualifiée tentant d'échapper à la concurrence du marché du travail des métropoles. Les villes moyennes en tant qu'espace urbain permettent aussi l'accès à un certain nombre de services et de commerces (Bochet et al., 2004). L'accès à ces services dans les villes moyennes tels que les soins de santé, l'éducation et les commodités impactent la population et les entreprises locales mais jouent également un rôle important dans les liens rurauxurbains puisqu'elles servent également la population environnante localisée dans l'hinterland de la ville (Satterthwaite et Tacoli, 2003). 
En synthèse, parmi les avantages urbains dont peuvent tirer parti les entreprises agroalimentaires localisées en ville moyenne, les facteurs suivants sont susceptibles d'émerger : l'accès à un marché du travail local plus important et plus efficient, l'accès à des infrastructures de transport et de logistique, l'accès à des services de base et l'existence d'effets d'agglomération dus à la présence de districts industriels ou de clusters.

\section{Les zones rurales et leurs spécificités}

À l'opposé des zones métropolitaines, les zones rurales se caractérisent par un faible degré d'agglomération. La figure centrale du monde rural aujourd'hui est représentée par la campagne comme cadre de vie (Bigot et Hatchuel, 2001 ; Perrier-Cornet et Hervieu, 2002). Le monde rural apparaît comme un antidote à la ville, traduisant une évolution du style de vie des individus (Mora et al., 2012). La ruralité n'est donc plus appréhendée comme un unique espace de production agricole mais comme un espace cadre de vie. Cet aspect des zones rurales est d'autant plus fort que la montée des préoccupations environnementales et le rejet de la grande ville entraînent un désir de retour à un cadre de vie naturel d'idylle rurale (Bailly et Bourdeau-Lepage, 2011). Les villes moyennes permettent de répondre à ces conditions dans les régions à prédominance rurale en attirant des personnes à la recherche d'un mode de vie spécifique (Delfmann et al., 2014). Ainsi, les évolutions des modes de transport et de communication permettent de compenser les aspects négatifs en faisant de l'espace rural un lieu d'épanouissement et de liberté (Mora et al., 2012).

Les zones rurales permettent également d'accéder à du foncier abondant et abordable (Lecat, 2004). Elles permettent aussi de pallier les externalités négatives des zones métropolitaines-qui par ailleurs renforcent les avantages et la valeur des aménités des zones rurales (Blanc, 1997).
Dans le cas particulier d'entreprises du secteur agroalimentaire, certaines caractéristiques rurales peuvent concourir à leur avantage concurrentiel. La littérature sur le développement régional souligne l'importance des spécificités locales dans les zones faiblement peuplées telles que les commodités, les intrants ou les ressources (McGranahan et al., 2011; Naldi et al., 2015). De plus, les entreprises agroalimentaires sont situées à proximité de la ressource agricole (Triboulet et al., 2015) et peuvent aussi accéder à une maind'œuvre agricole. Ménage (2011) met en évidence un effet «ville moyenne» au moment de la création et de l'implantation des entreprises lié à des marchés locaux ou des liens interpersonnels. Cet effet sera renforcé dans le secteur agricole où la localisation dans un espace rural permet d'accéder à un processus de valorisation du territoire dans un espace rural (Couzinet et al., 2002) malgré des différences entre les filières de production agricole (Torre et Pham, 2015).

Il est ainsi possible de conclure que, parmi les avantages dont peuvent bénéficier les entreprises agroalimentaires localisées en villes moyennes, se dessinent un cadre de vie agréable et proche de la nature, un coût du foncier moindre, mais aussi, plus particulièrement dans le secteur de l'agroalimentaire, une proximité avec la ressource agricole, l'accès à une maind'œuvre agricole qualifiée et à un processus de valorisation du territoire.

Afin de tester l'ensemble des hypothèses formulées précédemment, la méthodologie mise en œuvre est détaillée dans la section suivante.

\section{Méthodologie et données}

Les hypothèses proposées sont testées grâce à des études de cas construites à partir d'entretiens semi-directifs. Dix entreprises localisées dans cinq villes moyennes de la région Occitanie ont été enquêtées. 
En effet, Demazière (2017) souligne que par rapport aux travaux sur les dynamiques métropolitaines, les travaux étudiant l'encastrement des activités économiques dans les structures sociales (Granovetter, 1985) par des méthodes qualitatives semblent plus adaptés pour étudier les villes moyennes, à l'inverse des approches focalisées sur les effets d'agglomération cherchant à expliquer la croissance des villes (Carrier et Demazière, 2012). Les données ont été analysées grâce au logiciel NVivo. Après une présentation du corpus d'entretiens, la démarche méthodologique de l'étude et les caractéristiques du logiciel seront détaillées.

\section{Les données : sélection des villes moyennes et des cas d'entreprises innovantes}

L'article entend dégager les facteurs de différenciation des villes moyennes à travers les déterminants de la localisation des entreprises agroalimentaires dans ces espaces. La démarche méthodologique a consisté dans un premier temps à sélectionner un ensemble de cinq villes moyennes localisées en Occitanie. Ces villes ont été sélectionnées selon un double critère de taille - une aire urbaine dont le pôle d'emploi à la population de sa ville centre comprise entre 20000 et 100000 habitants (Floch et Morel, 2001) - et de fonction - critères géographiques et fonctionnels: distance aux métropoles, présence de services de base et de centres de décision administratifs et connectivité de la ville (insertion dans les réseaux de transport, accès aux TIC). Ainsi, Cahors, Rodez, Montauban, Alès et Béziers ont été retenues.

Le tableau 1 présente une synthèse des caractéristiques de chacune de ces villes permettant d'expliquer les critères de choix abordés dans la partie ci-dessus.

\section{Tableau 1. Caractéristiques de villes moyennes sélectionnées}

\begin{tabular}{|c|c|c|c|c|c|c|}
\hline Ville & & Cahors & Rodez & Montauban & Alès & Béziers \\
\hline \multirow{3}{*}{$\begin{array}{l}\text { Données socio- } \\
\text { économiques }\end{array}$} & Taille $^{1}$ & 44255 & 85181 & 107436 & 114137 & 171010 \\
\hline & Surface (en km²) & 715,9 & 1066 & 795,80 & 653,90 & 708,40 \\
\hline & Fonctions urbaines & Préfecture & Préfecture & Préfecture & $\begin{array}{l}\text { Sous- } \\
\text { préfecture }\end{array}$ & $\begin{array}{l}\text { Sous- } \\
\text { préfecture }\end{array}$ \\
\hline \multirow[t]{4}{*}{$\begin{array}{l}\text { Environnement } \\
\text { économique }\end{array}$} & $\begin{array}{l}\text { Nombre } \\
\text { d'établissements }^{2}\end{array}$ & 4941 & 9292 & 11296 & 11257 & 20010 \\
\hline & $\begin{array}{l}\text { Nombre } \\
\text { d'établissements IAA }{ }^{3}\end{array}$ & 17 & 36 & 21 & 22 & 31 \\
\hline & Taux de chômage ${ }^{4}$ & 13.9 & 13.9 & 14.20 & 19.30 & 18.60 \\
\hline & $\begin{array}{l}\text { Présence d'une chambre } \\
\text { de commerce }\end{array}$ & Oui & Oui & Oui & Oui & Oui \\
\hline \multirow{4}{*}{$\begin{array}{l}\text { Infrastructures } \\
\text { de transport }\end{array}$} & Présence d'un aéroport & Non & Oui & Non & Non & Oui \\
\hline & Présence d'une gare TGV & Non & Non & Oui & Non & Oui \\
\hline & $\begin{array}{l}\text { Proximité avec } \\
\text { l'autoroute }\end{array}$ & Oui & Non & Oui & Non & Oui \\
\hline & $\begin{array}{l}\text { Distance aux métropoles } \\
\text { (et temps de transport) }\end{array}$ & $\begin{array}{c}\text { Toulouse } \\
115 \mathrm{~km} \\
(1 \mathrm{~h} 25)\end{array}$ & $\begin{array}{c}\text { Toulouse } \\
135 \mathrm{~km}(1 \mathrm{~h} 48) \\
\text { Montpellier : } \\
160 \mathrm{~km}(2 \mathrm{~h} 30)\end{array}$ & $\begin{array}{c}\text { Toulouse } \\
60 \mathrm{~km} \\
(0 \mathrm{~h} 45)\end{array}$ & $\begin{array}{c}\text { Montpellier } \\
95 \mathrm{~km} \\
(1 \mathrm{~h} 30)\end{array}$ & $\begin{array}{c}\text { Montpellier } \\
87 \mathrm{~km} \\
(1 \mathrm{~h} 00)\end{array}$ \\
\hline
\end{tabular}

Source : les auteurs.

1. Taille de l'aire urbaine: population de l'aire urbaine, INSEE (2013).

3. DADS (2012).

2. Nombre d'établissements au 31 décembre 2014, 4. Taux de chômage des 15-64 ans, INSEE INSEE. 
RECHERCHE

Geoffroy LABROUCHE, Rachel LEVY

Tableau 2. Caractéristiques des cas d'entreprises étudiées

\begin{tabular}{|c|c|c|c|c|c|}
\hline Entreprises & Ville & Activité & $\begin{array}{c}\text { Taille } \\
\text { (nombre } \\
\text { d'employés) }\end{array}$ & $\begin{array}{l}\text { Date de création } \\
\text { de l'entreprise }\end{array}$ & $\begin{array}{c}\text { Fonction de la } \\
\text { personne interviewée }\end{array}$ \\
\hline E1 & Alès & $\begin{array}{c}\text { Négoce et élevage } \\
\text { de vin }\end{array}$ & 2 & 2008 & Dirigeant \\
\hline E2 & Béziers & Production de bière & 5 & 2016 & Associé \\
\hline E 3 & Cahors & $\begin{array}{l}\text { Fabrication de } \\
\text { meringue }\end{array}$ & 17 & 1983 & Dirigeant \\
\hline E4 & Cahors & Produits régionaux & 10 & 1996 & Dirigeant \\
\hline E5 & Cahors & Produits truffés & 10 & 1897 & Dirigeant \\
\hline E6 & Montauban & Aliment pour bétail & 6 & 1992 & Dirigeant \\
\hline E7 & Rodez & Production de viande & 300 & 1970 & Directeur industriel \\
\hline E8 & Rodez & Produits régionaux & 17 & 1988 & Dirigeant \\
\hline E9 & Rodez & Sélection variétale & 1000 & 1960 & $\begin{array}{c}\text { Directeur des affaires } \\
\text { scientifiques }\end{array}$ \\
\hline E10 & Rodez & Production de viande & 130 & 1994 & Responsable qualité \\
\hline
\end{tabular}

La seconde étape a consisté à répertorier les entreprises du secteur de l'agroalimentaire ${ }^{5}$ localisées dans les communes appartenant aux 5 aires urbaines sélectionnées. Finalement, 10 études de cas d'entreprises ${ }^{6}$ aux caractéristiques variées (tableau 2) ont été réalisées.

\section{Méthode de l'entretien semi-directif}

La récolte des données s'est faite par entretiens semi-directifs. Le choix d'une méthodologie qualitative est fondé sur le fait que les données sont particulièrement bien adaptées aux cas intégrés dans un contexte singulier-lié notamment aux caractéristiques de l'environnement. Elles permettent d'étudier la stratégie d'une entreprise de manière globale et dynamique et sont particulièrement indiquées lorsque la

5. Codes NAF 2008: C10 + C11 auxquels les boulangeries et les charcuteries ont été retranchées. 6. Un critère de choix important concernait le caractère innovant de ces entreprises. En effet, cette enquête fait partie d'un volet spécifique du PSDR4 sur l'innovation des entreprises agroalimentaires dans les villes moyennes. question concerne de nouveaux domaines théoriques, la compréhension de phénomènes peu étudiés et l'étude de variables non encore spécifiées ou testées (Glaser et Strauss, 1967 ; Shah et Corley, 2006 ; Yin, 2014). Ces méthodologies se concentrent sur les processus, l'expérimentation et fournissent un moyen de développer une compréhension des phénomènes complexes du point de vue de ceux qui les éprouvent (Miles et Huberman, 1991). Les études de cas qui suivent sont essentiellement fondées sur des données primaires issues d'entretiens semi-directifs?

Le guide d'entretien comprenait notamment des questions relatives à l'histoire de l'entreprise, son activité, ses marchés, ainsi qu'au parcours professionnel du dirigeant et des questions sur les avantages et des inconvénients d'être localisé dans une ville moyenne. Finalement, 10 entretiens dans 5 villes moyennes ont été réalisés

7. Ces données primaires ont été complétées par des données secondaires issues d'Internet et de la presse. 
entre le 20 février et le 4 septembre 2017 et ont ensuite été retranscrits mot à mot puis codés à l'aide du logiciel Nvivo.

\section{Traitement des données avec NVivo}

Les retranscriptions des entretiens ont été traitées grâce au logiciel Nvivo. Contrairement aux logiciels d'analyse lexicale, le logiciel Nvivo n'a pas pour objectif principal de faire de l'analyse textuelle à partir de recherche de mots mais d'aider le chercheur dans sa démarche d'analyse qualitative thématique d'un corpus de documents, en lui fournissant un outil de classement et d'organisation d'informations puis des possibilités informatiques de recherche (Bazeley et Jackson, 2013). Dans le cas du logiciel Nvivo, la démarche consiste à opérer une décontextualisation-recontextualisation du corpus à analyser (Deschenaux et Bourdon, 2005). Il s'agit en premier lieu de construire des catégories thématiques regroupant des segments de textes indépendants - autrement dit de coder le corpus, puis en deuxième lieu d'établir des relations entre les codes afin de créer un ensemble porteur de sens. Nvivo permet ainsi d'organiser cette décontextualisation en construisant une nouvelle structure. Le codage de données dans le logiciel Nvivo se fait donc à partir de plusieurs catégories d'éléments principaux : le cas, le nœud, la source, la référence. Ces différents éléments et leurs applications dans le cadre de notre étude sont définis dans le tableau 3 suivant.

L'analyse thématique des entretiens commence donc à l'étape du codage, c'està-dire à l'instant où le chercheur affecte les différentes séquences de texte aux nœuds entrés dans le logiciel (Thietart, 2014). La détermination des nœuds dépend de la démarche méthodologique entreprise par le chercheur. Dans notre étude, le codage a été réalisé en deux étapes grâce à une première phase de codage a priori puis une seconde phase de codage a posteriori (Allard-Poesi, 2003 ; Avenier et Thomas, 2015). Le but étant de créer un processus de conceptualisation progressif en distinguant un premier codage proche des hypothèses et un deuxième codage davantage tourné vers l'abstraction (Miles et al., 2013 ; Gioia et al., 2013).

La première codification cherchait à identifier les verbatim relatifs aux avantages et inconvénients liés au rural, à l'urbain et aux villes moyennes. Ainsi, cinq nœuds ont été créés : avantage, inconvénient, rural, urbain et ville moyenne. Les

\section{Tableau 3. Types de catégories d'éléments d'analyse à partir le logiciel Nvivo}

\begin{tabular}{|c|c|c|}
\hline $\begin{array}{l}\text { Type } \\
\text { d'éléments }\end{array}$ & Définition & $\begin{array}{l}\text { Nombre et type de catégories } \\
\text { dans le cadre de notre analyse }\end{array}$ \\
\hline Cas & Unité d'analyse : chaque cas est composé de sources & 5 cas de villes moyennes \\
\hline Sources & Corpus de documents importés dans le logiciel & $\begin{array}{l}10 \text { sources : retranscriptions } \\
\text { d'entretiens avec } 10 \text { entreprises }\end{array}$ \\
\hline Nœuds & $\begin{array}{l}\text { Le nœud représente le code. Chaque nœud est défini a } \\
\text { priori par les analystes soit à partir de la littérature soit } \\
\text { en émergeant au fur et à mesure de l'analyse. Chaque } \\
\text { nœud correspond ainsi à un thème précis, une idée ou une } \\
\text { catégorie. Le codage à partir de « nœuds » permet ainsi de } \\
\text { trier et de générer de la cohérence dans le jeu de données } \\
\text { et ensuite de visualiser comment ces dernières peuvent } \\
\text { permettre de répondre à la question de recherche. }\end{array}$ & 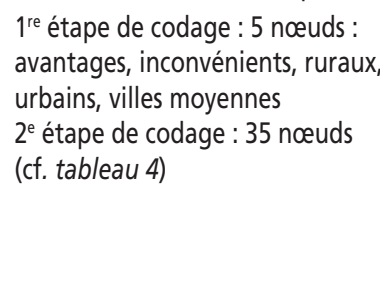 \\
\hline Références & $\begin{array}{l}\text { Verbatim (segment de texte) encodé dans un ou plusieurs } \\
\text { nœuds par l'analyste. }\end{array}$ & 246 références au total \\
\hline
\end{tabular}


entretiens ont été codés individuellement par chacun des deux coauteurs de l'article. Après validation du codage, le taux intercodeur moyen était égal à 95,70 \% (Bazeley et Jackson, 2013), autorisant la fusion des codages. NVivo permet à ce stade de l'analyse d'effectuer des requêtes afin d'aider le chercheur à unifier les concepts. Dans le cas de cet article, il a été possible de générer une requête afin d'associer les nœuds « avantages » et « inconvénients » aux nœuds correspondants aux trois types de territoires : « urbain », « rural » et « ville moyenne ». Les résultats de cette requête ont permis de générer deuxième codage davantage tourné vers l'abstraction (Miles et al., 2013 ; Gioia et al., 2013), a posteriori et émergeant des données. Ce codage, présenté dans le tableau 4 concernait les caractéristiques des trois types de territoire. Le corpus a ensuite été recodé séparément selon ces nouveaux nœuds puis comparé. Cette seconde étape de codage a également été effectuée en double aveugle puis les codes ont été fusionnés avec un taux intercodeur moyen de $99,74 \%$ pour aboutir à un total de 246 références codées présentées dans le tableau 4. L'analyse de ce tableau, qui présente également le nombre de sources, permet ainsi de tester les hypothèses. Les résultats de l'article se fondent, en effet, sur le nombre de références en lien avec un avantage ou un inconvénient donné, complété par le nombre d'entreprises y faisant référence (nombre de sources). Ainsi, si un avantage spécifique est très cité dans les entretiens par beaucoup d'entreprises différentes, il est possible de considérer que cet avantage est important, même si cette méthode présente l'inconvénient d'attribuer un même poids à des facteurs d'importances potentiellement différentes.

\section{Résultats}

La présentation des résultats se fera en deux parties. La première partie présentera les principaux éléments qui caractérisent les villes moyennes et testera la première hypothèse. La deuxième partie étudiera plus précisément les cas de Rodez et Cahors, dans le but de dégager des facteurs de différenciation et de tester la seconde hypothèse.

\section{Avantages et inconvénients d'une localisation en ville moyenne}

Le tableau 4 synthétise les principaux résultats sur les facteurs de localisation dans les villes moyennes, issus de l'analyse NVivo.

Plusieurs éléments de codage très spécifiques à la catégorie «ville moyenne » (90 références) sont mis en évidence, ce qui conforte l'idée que la ville moyenne est une catégorie d'urbain spécifique distincte du périurbain. Les éléments de codage font également apparaître des facteurs de localisation des entreprises tant associés aux caractéristiques rurales (92 références) qu'urbaines (55 références) des villes moyennes ce qui amène à penser que la ville moyenne serait une catégorie se rapprochant plus du rural.

Les entretiens font apparaître que la localisation dans une ville moyenne est principalement motivée par les avantages qu'elle procure même si des inconvénients non négligeables sont présents (148 références à des avantages, 89 références d'inconvénients). Cette analyse exploratoire tend à confirmer - au moins partiellement - l'hypothèse $\mathrm{H} 1$ : la ville moyenne se caractérise par des avantages liés à la fois à l'urbain et au rural. Cependant, les avantages ruraux sont plus présents et un certain nombre de critères spécifiques aux villes moyennes apparaissent-différents des précédents et peu évoqués dans la littérature.

\section{Avantages d'une localisation en ville moyenne}

Parmi les critères les plus fréquemment évoqués pour expliquer la localisation en ville moyenne, celui du cadre de vie proche de la nature est une caractéristique 
Tableau 4. Nombre de références et de sources des différents facteurs de localisation des entreprises agroalimentaires localisées en ville moyenne

\begin{tabular}{|c|c|c|c|c|c|c|c|}
\hline \multirow{4}{*}{$\begin{array}{l}\text { Catégorie } \\
\begin{array}{l}\text { Avantages } \\
\text { ruraux }\end{array}\end{array}$} & \multirow[b]{2}{*}{ Détail } & Réf. & Sour. & Réf. & Sour. & Réf. & Sour. \\
\hline & & \multicolumn{2}{|c|}{$\begin{array}{c}\text { Total des } \\
5 \text { villes } \\
\text { moyennes } \\
\end{array}$} & \multicolumn{2}{|c|}{ Rodez } & \multicolumn{2}{|c|}{ Cahors } \\
\hline & Cadre de vie agréable - Nature & 14 & 7 & 5 & 3 & 6 & 2 \\
\hline & Coût du foncier faible & 7 & 5 & 0 & 0 & 3 & 3 \\
\hline & $\begin{array}{l}\text { Proximité avec la ressource } \\
\text { et avec les fournisseurs }\end{array}$ & 7 & 4 & 4 & 2 & 0 & 0 \\
\hline & $\begin{array}{l}\text { Accès à une main-d'œuvre qualifiée } \\
\text { agricole }\end{array}$ & 5 & 4 & 0 & 0 & 0 & 0 \\
\hline & $\begin{array}{l}\text { Argument marketing et valorisation } \\
\text { du territoire }\end{array}$ & 2 & 2 & 0 & 0 & 1 & 1 \\
\hline & $\begin{array}{l}\text { Diminution des temps de transport } \\
\text { domicile-travail }\end{array}$ & 4 & 3 & 4 & 3 & 0 & 0 \\
\hline & Coût de la vie faible & 3 & 2 & 0 & 0 & 0 & 0 \\
\hline & Sous-total & 42 & N/A & 13 & N/A & 16 & N/A \\
\hline \multirow{8}{*}{$\begin{array}{l}\text { Avantages } \\
\text { urbains }\end{array}$} & Accès à un bassin de main-d'œuvre & 3 & 2 & 1 & 1 & 2 & 1 \\
\hline & $\begin{array}{l}\text { Accès aux transports - Autoroutes, } \\
\text { TGV, aéroport }\end{array}$ & 8 & 7 & 5 & 4 & 1 & 1 \\
\hline & Plateforme logistique & 12 & 6 & 4 & 3 & 5 & 1 \\
\hline & $\begin{array}{l}\text { Dynamisme économique } \\
\text { (Sous-traitants...) }\end{array}$ & 13 & 5 & 7 & 3 & 0 & 0 \\
\hline & Présence de services de base & 5 & 3 & 4 & 2 & 1 & 1 \\
\hline & Présence de services économiques & 5 & 4 & 3 & 2 & 0 & 0 \\
\hline & Accès à Internet haut-débit & 2 & 2 & 2 & 2 & 0 & 0 \\
\hline & Sous-total & 48 & N/A & 26 & N/A & 9 & $\mathrm{~N} / \mathrm{A}$ \\
\hline \multirow{7}{*}{$\begin{array}{l}\text { Avantages } \\
\text { Ville moyenne }\end{array}$} & Accès à des dispositifs d'aide spécifiques & 7 & 3 & 2 & 1 & 0 & 0 \\
\hline & Ville agréable à vivre & 15 & 8 & 6 & 3 & 5 & 2 \\
\hline & Identité spécifique & 15 & 6 & 4 & 3 & 6 & 2 \\
\hline & Politiques volontaristes & 5 & 2 & 5 & 2 & 0 & 0 \\
\hline & Proximité avec la ou les métropoles & 13 & 9 & 5 & 3 & 4 & 3 \\
\hline & Proximité avec l'université & 3 & 2 & 0 & 0 & 0 & 0 \\
\hline & Sous-total & 58 & N/A & 22 & N/A & 15 & N/A \\
\hline \multirow{11}{*}{$\begin{array}{l}\text { Inconvénients } \\
\text { ruraux }\end{array}$} & Difficulté de trouver de la main-d'œuvre & 8 & 4 & 5 & 2 & 3 & 2 \\
\hline & Difficultés logistiques & 6 & 5 & 2 & 2 & 3 & 2 \\
\hline & Manque de dynamisme économique & 2 & 1 & 0 & 0 & 2 & 1 \\
\hline & $\begin{array}{l}\text { Mauvais services aux entreprises - } \\
\text { réseaux peu adaptés }\end{array}$ & 4 & 2 & 1 & 1 & 3 & 1 \\
\hline & Absence de recherche scientifique & 4 & 3 & 2 & 1 & 1 & 1 \\
\hline & Difficultés déplacement individuel & 8 & 4 & 6 & 2 & 2 & 2 \\
\hline & Faible salaire & 3 & 1 & 3 & 1 & 0 & 0 \\
\hline & Isolement & 13 & 6 & 7 & 4 & 6 & 2 \\
\hline & Pas de vie culturelle - loisirs & 5 & 3 & 1 & 1 & 1 & 1 \\
\hline & Pas d'emploi pour le conjoint & 3 & 3 & 2 & 2 & 1 & 1 \\
\hline & Sous-total & 56 & N/A & 29 & $\mathrm{~N} / \mathrm{A}$ & 22 & N/A \\
\hline
\end{tabular}




\begin{tabular}{|c|c|c|c|c|c|c|c|}
\hline \multirow[b]{2}{*}{ Catégorie } & \multirow[b]{2}{*}{ Détail } & Réf. & Sour. & Réf. & Sour. & Réf. & Sour. \\
\hline & & \multicolumn{2}{|c|}{$\begin{array}{c}\text { Total des } \\
5 \text { villes } \\
\text { moyennes }\end{array}$} & \multicolumn{2}{|c|}{ Rodez } & \multicolumn{2}{|c|}{ Cahors } \\
\hline \multirow{4}{*}{$\begin{array}{l}\text { Inconvénients } \\
\text { urbains }\end{array}$} & Temps de transport importants & 2 & 2 & 2 & 2 & 0 & 0 \\
\hline & Prix du foncier élevé & 2 & 2 & 0 & 0 & 1 & 1 \\
\hline & Taxes élevées (foncier, aménagement) & 3 & 3 & 0 & 0 & 2 & 1 \\
\hline & Sous-total & 7 & $\mathrm{~N} / \mathrm{A}$ & 2 & $\mathrm{~N} / \mathrm{A}$ & 3 & $\mathrm{~N} / \mathrm{A}$ \\
\hline \multirow[t]{4}{*}{$\begin{array}{l}\text { Inconvénients } \\
\text { Ville moyenne }\end{array}$} & $\begin{array}{l}\text { Manque de dynamisme économique } \\
\text { et politique }\end{array}$ & 15 & 5 & 2 & 1 & 12 & 3 \\
\hline & Mauvaise image de la ville & 7 & 4 & 2 & 2 & 0 & 0 \\
\hline & Ville qui meurt & 10 & 4 & 1 & 1 & 7 & 2 \\
\hline & Sous-total & 32 & $\mathrm{~N} / \mathrm{A}$ & 5 & $\mathrm{~N} / \mathrm{A}$ & 19 & $\mathrm{~N} / \mathrm{A}$ \\
\hline \multirow[t]{5}{*}{ Totaux } & Total des avantages & 148 & $\mathrm{~N} / \mathrm{A}$ & 61 & $\mathrm{~N} / \mathrm{A}$ & 37 & $\mathrm{~N} / \mathrm{A}$ \\
\hline & Total des inconvénients & 89 & $\mathrm{~N} / \mathrm{A}$ & 32 & $\mathrm{~N} / \mathrm{A}$ & 44 & $\mathrm{~N} / \mathrm{A}$ \\
\hline & Total des arguments ruraux & 92 & $\mathrm{~N} / \mathrm{A}$ & 42 & $\mathrm{~N} / \mathrm{A}$ & 35 & $\mathrm{~N} / \mathrm{A}$ \\
\hline & Total des arguments urbains & 55 & $\mathrm{~N} / \mathrm{A}$ & 28 & $\mathrm{~N} / \mathrm{A}$ & 12 & $\mathrm{~N} / \mathrm{A}$ \\
\hline & Total des arguments villes moyennes & 90 & $\mathrm{~N} / \mathrm{A}$ & 27 & $\mathrm{~N} / \mathrm{A}$ & 34 & $\mathrm{~N} / \mathrm{A}$ \\
\hline
\end{tabular}

forte (14 références issues de 7 sources). La localisation des entreprises agroalimentaires en ville moyenne s'explique en grande partie par la volonté de profiter d'un cadre de vie spécifique différencié de celui des métropoles. Ces chefs d'entreprises précisent: "Vous n'avez qu'à regarder par la fenêtre, l'environnement est extraordinaire » [E4], et un autre d'ajouter : «Le soir on rentre et on regarde les coccinelles traverser le jardin » [E10].

En complément de ces arguments liés aux choix individuels des chefs d'entreprises et liés à la facette rurale de la ville moyenne, se retrouvent des arguments liés au cadre urbain, en particulier les services de base ( 5 références issues de 3 sources). Se localiser dans une ville moyenne permet donc de concilier un désir de nature et de vivre à la campagne tout en accédant aux commodités offertes par la ville (Perrier-Cornet et Hervieu, 2002).

Enfin, les éléments liés au cadre de vie sont complétés par des caractéristiques propres aux villes moyennes, par exemple le caractère agréable à vivre de la ville (15 références issues de 8 sources) et l'existence d'une identité spécifique de la ville (15 références issues de 6 sources).

Un deuxième ensemble de critères concerne les éléments facilitateurs de l'activité économique: l'existence d'un dynamisme économique et la possibilité de bénéficier d'effets d'agglomérations (13 références dans 5 sources), la proximité avec les plateformes logistiques (12 références dans 6 sources), l'accès à des réseaux de transports (8 références dans 7 sources), la présence de services économiques ( 5 références dans 4 sources) et dans une moindre mesure l'accès à un bassin de main-d'œuvre (3 références dans 2 sources). Ces critères relèvent principalement des caractéristiques des métropoles. En effet, les problématiques de transport et de logistique sont fondamentales pour les personnes et les entreprises et renvoient à la notion de connectivité externe de la ville 
moyenne (Nadou, 2010). Une entreprise souligne : «On n'a aucun souci de transport, on est bien desservi par les messageries. C'est important parce que les gens stockent peu et nous on a du produit en permanence, on n'est jamais en rupture de stock. » [E6].

Ces éléments qui se rapportent aux métropoles sont complétés par des arguments qui sont plus spécifiques à une localisation dans une ville moyenne tels que la proximité avec les métropoles (13 références dans 9 sources), la possibilité d'accéder à des aides spécifiques (7 références dans 3 sources) et la présence de politiques volontaristes de la ville envers les entreprises (5 références dans 2 sources).

Un troisième ensemble d'arguments concerne les spécificités propres aux entreprises agroalimentaires et notamment une localisation proche des espaces ruraux. La proximité à la ressource agricole est importante (7 références dans 4 sources) et l'accès à une main-d'œuvre agricole qualifiée est aussi évoqué (5 références dans 4 sources) ainsi que l'existence d'un processus de valorisation des produits du territoire rural (2 références dans 2 sources, par exemple : «Fabrication artisanale dans le Lot, ça fait plus terroir » [E3] confirmant les analyses de Barre (1999) qui a montré que dans le secteur de la production de viande contrairement aux grands groupes qui adoptent des stratégies de volumes et de différenciation, les PME vont rechercher des micromarchés et s'ancrer dans des systèmes productifs locaux.

Enfin, des critères d'ordre financier ressortent comme le faible coût du foncier (7 références dans 5 sources) ou le moindre coût de la vie (3 références dans 2 sources).

\section{Inconvénients d'une localisation} en ville moyenne

Si la localisation en ville moyenne possède beaucoup d'avantages, un certain nombre d'inconvénients (89 références à des inconvénients vs 148 références à des avantages) sont soulevés relativisant la validation de l'hypothèse $1^{8}$.

Ainsi, le principal inconvénient évoqué fait référence à un débat qui est au cœur de la problématique des villes moyennes : celui de leur dynamisme. Le manque de dynamique économique et politique (15 références dans 5 sources), la mort lente des centres-villes et la mauvaise image de la ville ( 7 références dans 4 sources) sont mis en avant.

Les inconvénients spécifiques aux territoires ruraux sont également évoqués : manque de vie culturelle et de loisirs (5 références par 3 sources), difficulté à trouver un emploi pour les conjoints ( 3 références par 3 sources) ou encore niveau de salaire faible (3 références par une seule entreprise). Un inconvénient ressort cependant de manière plus importante, il s'agit de l'isolement des entreprises (13 références par 6 sources). Si cet inconvénient est cité un grand nombre de fois par les entreprises, cela s'explique par un même élément de langage qui évoque l'isolement de la ville moyenne qui ne dispose pas d'infrastructure de transport à l'instar de E4 : «On était complètement isolés, mais la communication ça passe par des voies de communications. Nous on n'a pas le $T G V$, on n'a pas d'aéroport, l'aéroport il a été fait à côté de Brive, à juste titre sûrement, mais on est pris entre Brive d'un côté, Montauban et Toulouse de l'autre ». L'isolement évoque aussi un éloignement des entreprises qui se retrouvent enfermées dans leurs territoires comme E9 qui « a tendance à se trouver isolé à Rodez ».

Le tableau 4 fait aussi apparaître d' autres types d'inconvénients ponctuellement cités

8. Pour renforcer ces résultats, dans l'ensemble du corpus, le terme avantage(s) apparaît 28 fois alors que le terme inconvénient(s) n'apparaît que 15 fois. 
et qui semblent très spécifiques à quelques entreprises, faisant notamment référence à des arguments présentés comme des avantages pour la plupart des entreprises. Il semble ainsi que les villes moyennes ne regroupent pas une catégorie homogène de villes, mais qu'au-delà des traits caractéristiques communs qui les définissent, elles se différencient par des contextes particuliers qui ne peuvent être mis en avant par un simple comptage des références sur l'ensemble des entretiens réalisés. La partie suivante complète cette analyse en étudiant les facteurs de différenciation.

\section{Facteurs de différenciation des villes moyennes. Les cas de Cahors et Rodez}

La mise en évidence des facteurs de différenciation passe par une étude approfondie de deux villes qui semblent avoir développé des trajectoires d'évolution opposées : Cahors et Rodez. Ces deux villes sont situées dans des zones considérées par l'OCDE comme des zones rurales majoritairement isolées (Brezzi et al., 2011) et représentent le centre administratif de deux départements ruraux. Cette caractéristique de localisation se traduit notamment par un isolement par rapport aux deux métropoles régionales - Toulouse et Montpellier - ce qui limite les possibles effets de rayonnement des métropoles sur les caractéristiques des villes. Cependant, les deux villes se distinguent par leur taille et leur superficie, par leur dynamisme économique et leur environnement géographique (tableau 2). Cahors est d'une taille plus petite que Rodez et se caractérise par une localisation sur l'axe autoroutier Paris-Toulouse tandis que Rodez se situe dans un département au relief accidenté et difficile d'accès. Ces deux études de cas ont ainsi pour objectif de dégager des facteurs différenciants dus à la fois à leur contexte sociohistorique et géographique, mais surtout à leur condition de ville moyenne.
Les données du tableau 4 permettent de confirmer l'hypothèse $\mathrm{H} 2$ et les éléments évoqués dans la sous-partie précédente : l'existence de la catégorie ville moyenne. Afin de mieux les cerner, il est dans un premier temps possible d'observer un certain nombre de points communs qui expliquent que des entreprises restent ou viennent se localiser dans ces espaces. En particulier, le cadre et la qualité de vie proches de la nature sont mis en avant (5 références dans 3 entreprises à Rodez et 6 références dans 2 entreprises à Cahors). Ces villes sont agréables à vivre (6 références dans 3 entreprises à Rodez et 5 références dans 2 entreprises à Cahors) et ont développé une identité spécifique à la ville moyenne (4 références dans 3 entreprises à Rodez et 6 références dans 2 entreprises à Cahors). Ces arguments renforcent l'idée qu'il existe un cadre de vie non pas rural mais spécifique à la ville moyenne.

Les critères liés aux activités économiques sont également présents comme la proximité avec des plateformes et des équipements logistiques (4 références dans 3 entreprises à Rodez et 5 références dans 1 entreprise à Cahors), la proximité avec Toulouse ou Montpellier (5 références dans 3 entreprises à Rodez et 4 références dans 3 entreprises à Cahors) et dans une moindre mesure l'accès à un bassin de main-d'œuvre (souligné par 1 entreprise dans chaque ville). Enfin, les inconvénients des zones rurales ressortent également de la même manière dans les deux villes: manque de vie culturelle et de loisirs (1 entreprise dans chaque ville), difficulté à trouver un emploi pour le conjoint ( 2 entreprises à Rodez et 1 à Cahors) et isolement (4 entreprises à Rodez et 2 à Cahors). Ces arguments : un cadre de vie agréable à proximité de la nature, une proximité avec les métropoles et un accès aux transports, malgré un isolement et un manque de vie culturel semblent caractériser les villes moyennes. 
Malgré ces points communs entre Cahors et Rodez, se dégagent des critères de différenciation. En particulier, Rodez apparaît comme une ville moyenne beaucoup plus attractive notamment en raison de la présence de services de base, mais aussi de la présence de services économiques actifs qui apparaissent comme inefficaces sur Cahors. Parmi les services de base mentionnés par les entreprises ruthénoises, l'accès à Internet haut-débit ressort ainsi que la possibilité d'accéder à des dispositifs d'aide spécifiques. Rodez se distingue aussi de Cahors par l'accès aux réseaux de transport et la proximité à la ressource et aux fournisseurs. Il semble donc que parmi les critères de différenciation permettant d'expliquer les divergences de trajectoires entre villes moyennes la présence de services aux entreprises et l'accès facilité aux transports et aux plateformes logistiques jouent un rôle important pour les entreprises.

Le principal point de divergence entre les deux villes est observable au niveau de leur dynamisme économique perçu et des politiques publiques volontaristes de soutien aux entreprises. Le manque de dynamisme économique et politique apparaît comme un inconvénient majeur pour les entreprises cadurciennes (12 références par 3 entreprises sur à Cahors) qui considèrent Cahors comme une ville qui meurt (7 références par 2 entreprises). Ces premiers constats permettent de souligner le rôle essentiel de l'environnement institutionnel dans le développement des entreprises du secteur agroalimentaire de ces villes. En effet, ces dernières ont une politique différenciée de soutien aux entreprises qui semble générer des disparités.

À Rodez, le dynamisme économique s'explique par la présence de grandes entreprises qui structurent le tissu économique. La présence de ces grandes entreprises semble essentielle dans le maillage économique de la ville : "Que ce soit ça, les laiteries, la maison Malrieux qui a été reprise par des groupes... ça a créé un tissu économique, ça a fait venir des gens, ça a généré du bâti... » [E8]. Le dynamisme économique du territoire s'explique également par la présence de petites entreprises qui complètent le maillage, en particulier celles qui jouent le rôle de fournisseur: «On veut garder ce maillage et que tout le monde puisse garder son travail ici. Ici, il y a des grosses entreprises, mais je pense que c'est le maillage des toutes petites entreprises qui est important dans la dynamique » [E10].

Ce dynamisme économique ruthénois s'explique également par les politiques volontaristes d'aide aux entreprises qui permettent d'impulser la dynamique : «Peut-être que c'est plus soutenu économiquement aussi pour retenir les gens sur le territoire » [E8]. À l'opposé de l'exemple ruthénois, les entreprises cadurciennes soulignent le manque de dynamisme économique sur le territoire, qui viendrait d'une faiblesse dans les dispositifs de soutien aux entreprises malgré un fort potentiel : "C'est une ville qui $a d u$ potentiel mais qui n'a jamais exploité un vrai potentiel »[E4].

Cahors est également qualifiée par ses entreprises de «ville qui meurt», soulignant le débat sur les dynamiques de ces villes : «Il y a deux-trois affaires qui vivent à Cahors mais il n'y a pas de cour de vie quoi alors qu'il y a une vraie richesse... » [E4], un autre chef d'entreprise ajoute: «Oui, parmi les inconvénients, c'est qu'on fait partie des villes moyennes qui meurent. Vous parliez d'image de marque, l'image de marque elle serait portée si Cahors et son centre étaient attractifs. Un centre vivant et non pas un centre mort $\gg$ [E5].

Cependant, en dépit de ces points négatifs, Cahors dispose de quelques avantages comparatifs par rapport à Rodez, notamment en termes de coût du foncier et d'une main-d'œuvre qui semble plus qualifiée 
par rapport aux entreprises ruthénoises qui rencontrent des difficultés pour attirer et trouver de la main-d'œuvre.

Les exemples respectifs de Rodez et de Cahors confirment donc bien qu'il existe un certain nombre de différences entre les territoires qui appartiennent à la catégorie des villes moyennes.

$$
\begin{gathered}
* \\
* \quad *
\end{gathered}
$$

Les résultats confirment au moins partiellement, que la catégorie ville moyenne est une catégorie à part entière avec comme caractéristique principale de tirer parti des avantages urbains et ruraux sans en subir tous les inconvénients. L'hypothèse 1 est validée même si ce résultat doit être nuancé. En effet, la localisation d'entreprises agroalimentaires en ville moyenne semble présenter des avantages liés à la proximité de l'espace rural ainsi qu'à une qualité de vie spécifique qui n'existe pas dans les territoires métropolitains. Cette proximité au rural, positivement valorisée par les acteurs dans leur cadre de vie, est complétée par une série de fonctions propres aux espaces urbains qui caractérisent la ville moyenne et qui permettent aux entreprises de se développer grâce à la connectivité de la ville via les réseaux de transport et la proximité aux infrastructures logistiques.

Les résultats font apparaître des caractéristiques communes et spécifiques aux villes moyennes. Ce sont des caractéristiques positives, comme la proximité avec les grandes métropoles, et négatives, comme l'isolement et la perte de dynamisme. La catégorie ville moyenne doit donc être considérée comme une catégorie urbaine spécifique et différente de la métropole et du périurbain.

D'autre part, l'hypothèse 2 est également validée. Il existe au sein des villes moyennes des critères de différenciation qui expliquent des différences de dynamisme économique et de critères de localisation des firmes agroalimentaires. Ainsi, certains inconvénients présentés pour une catégorie de ville apparaissent aussi comme des avantages pour d'autres villes, avec par exemple une opposition très forte entre Rodez et Cahors. Plusieurs facteurs de différenciation ont émergé des études de cas pour expliquer ces différences entre villes moyennes : le dynamisme économique et le volontarisme des politiques et des organismes d'aide aux entreprises permettant d'aider les entreprises à se développer. Le développement des centres-villes et des aménités à destination des habitants explique aussi le développement inégal de ces villes moyennes. Il est donc nécessaire de mettre en place des politiques de développement des centresvilles à l'image du projet « actions cœur de villes » mis en place par le gouvernement français en 2018. De plus, il apparaît que le développement de ces villes dépend de l'émergence et de la consolidation d'un maillage d'entreprises de tailles moyennes connectées à l'environnement direct de la ville moyenne, mais aussi avec les métropoles localisées à proximité de ces villes moyennes. Ce développement ne peut se faire que par des politiques volontaristes et ambitieuses.

En termes méthodologiques, ce travail a permis de mettre en avant les apports d'une méthodologie d'étude de cas fondée sur le logiciel de traitement de données Nvivo qui a permis de mettre en avant les caractéristiques des villes moyennes. Cependant, ce travail ne constitue qu'une première étape dans la construction de l'objet « ville moyenne » et comporte des limites. En effet, ce travail est issu d'une analyse qualitative fondée sur un échantillon limité de 10 entreprises localisées dans des espaces spécifiques et uniquement dans le secteur agroalimentaire, un secteur très dépendant de la localisation géographique des entreprises. C'est pourquoi ce travail pourrait être complété par une étude statistique afin de généraliser ces 
résultats. Une comparaison avec d'autres secteurs et d'autres territoires serait également nécessaire. L'utilisation du logiciel NVivo et notamment le fait de distinguer les facteurs de caractérisation des villes moyennes par un simple comptage des références pourraient aussi être complétés par une analyse lexicale.

Cette recherche a bénéficié $d u$ soutien $d u$ Programme PSDR4 (projet REPROINNOV, 20162020), financé par I'INRA et la Région Occitanie 


\section{RÉFÉRENCES BIBLIOGRAPHIQUES}

Allard-Poesi F. (2003). Coder les données. In Giordano Y. (dir.), Conduire un projet de recherche, une perspective qualitative, Colombelles, Éditions EMS, pp. 245-290.

Audretsch D. B., Feldman M. P. (2004). Knowledge spillovers and the geography of innovation. Handbook of Regional and Urban Economics, Amsterdam, Elsevier, pp. 2713-2739.

Avenier M., Thomas C. (2015). Finding one's way around various methodological guidelines for doing rigorous case studies: A comparison of four epistemological frameworks. Systèmes d'information \& management, vol. $20, \mathrm{n}^{\circ} 1$, p. 61 .

Bailly A., Bourdeau-Lepage L. (2011). Concilier désir de nature et préservation de l'environnement: vers une urbanisation durable en France. Géographie, économie, société, vol. 1, $\mathrm{n}^{\circ} 13$, pp. 27-43.

Barre D. (1999). Survivre dans l'industrie des viandes : Les PME doivent se construire une rente. Viandes et produits carnés, vol. 20, $\mathrm{n}^{\circ} 4$, pp. 151-156.

Bazeley P., Jackson K. (2013). Qualitative Data Analysis with NVIVO. London, Sage.

Becattini G. (1992). Le district marshallien : une notion socio-économique. In Benko G., Lipietz A. (dir.), Les régions qui gagnent, Paris, PUF, pp. 37-39.

Bigot R., Hatchuel G. (2001). Les Français et l'espace rural, $C R E D O C$, juin.

Billings S. B., Johnson E. B. (2016). Agglomeration within an urban area. Journal of Urban Economics, $\mathrm{n}^{\circ} 91$, pp. 13-25.

Blanc M. (1997). La ruralité : diversité des approches. Économie rurale, $\mathrm{n}^{\circ} 242$, pp. 5-12.

Bochet B., Gay J.-B., Pini G. (2004). La ville dense et durable : un modèle européen pour la ville, Géoconfluences.

Bouba-Olga O., Ferru M., Guimond B. (2012). Organisation des activités et dynamiques territoriales : éléments d'analyse et application aux bassins de Cognac et de Châtellerault. Revue d'économie régionale \& urbaine, avril, $\mathrm{n}^{\circ} 2$, p. 173.
Bouba-Olga O., Grossetti M. (2015). La métropolisation, horizon indépassable de la croissance économique ? Revue de l'OFCE, vol. 143, $\mathrm{n}^{\circ}$, pp. 117-144.

Brezzi M., Dijkstra L., Ruiz V. (2011). OECD Extended Regional Typology, The Economic Performance of Remote Rural Regions. OECD Regional Development Working Papers, 2011/6.

Camagni R., Capello R., Caragliu A. (2015). The Rise of Second-Rank Cities: What Role for Agglomeration Economies? European Planning Studies, vol. 23, $\mathrm{n}^{\circ} 6$, pp. 1069-1089.

Campagnac-Ascher E. (2015). Économie de la connaissance. Une dynamique métropololitaine? Le Moniteur, CNDP-CRDP.

Carrier M., Demazière C. (2012). La socio-économie des villes petites et moyennes : questions théoriques et implications pour l'aménagement du territoire. Revue d'économie régionale \& urbaine, avril, $\mathrm{n}^{\circ} 2$, p. 135.

Charbonneau F., Lewis P., Manzagol C. (2003). Villes moyennes et mondialisation. Montréal, Trames Éditions.

Couzinet L., Frayssignes J., Pouzenc M., Simmoneaux J. (2002). La valorisation des aménités rurales comme stratégie d'acteurs : les exemples du Lot et de la filière fromagère AOC Rocamadour. Économie et sociétés, pp. 1465-1485.

Davezies L. (2010). La crise et nos territoires : premiers impacts. Paris, Caisse des dépôts, Institut CDC pour la recherche.

Davezies L., Pech T. (2014). La nouvelle question territoriale. Terra Nova.

Delfmann H., Koster S., McCann P., Dijk J. Van (2014). Population Change and New Firm Formation in Urban and Rural Regions. Regional Studies, vol. 48, $\mathrm{n}^{\circ} 6$, pp. 1034-1050.

Demazière C. (2014). Pourquoi et comment analyser les villes moyennes ? Un potentiel pour la recherche urbaine. Métropolitiques, janvier.

Demazière C. (2017). Le traitement des petites et moyennes villes par les études urbaines. 
Espaces et sociétés, vol. 168-169, $\mathrm{n}^{\circ} 1$, pp. 17.

Deschenaux F., Bourdon S. (2005). Introduction à l'analyse qualitative informatisée à l'aide du logiciel QSR Nvivo 2.0. Cahiers pédagogiques pour la recherche qualitative.

ESPON (2008). The Role of Small or MediumSized Towns. Final report.

Fearne A., María García Álvarez-Coque J., López-García Usach Mercedes T., García S. (2013). Innovative firms and the urban/ rural divide: the case of agro-food system, Management Decision, vol. 51, $\mathrm{n}^{\circ}$ 6, pp. 1293-1310.

Floch J.-M., Morel B. (2001). Panorama des villes moyennes. INSEE Documents de travail, H2011/01.

Florida R. (2002). The rise of the creative class. New York, Basic Books.

Frenken K., Boschma R. A. (2007). A theoretical framework for evolutionary economic geography: industrial dynamics and urban growth as a branching process. Journal of Economic Geography, vol. 7, $\mathrm{n}^{\circ}$ 5, pp. 635-649.

Gioia D. A., Corley K. G., Hamilton A. L. (2013). Seeking Qualitative Rigor in Inductive Research. Organizational Research Methods, vol. 16, n 1, pp. 15-31.

Glaser B., Strauss A. (1967). The discovery of grounded theory. Chicago, Aldine.

Granovetter M. (1985). Economic Action and Social Structure: The Problem of Embeddedness. American Journal of Sociology, vol. 91, n 3, pp. 481-510.

Guay L., Hamel P. (2004). Les villes contemporaines à la croisée des choix collectifs et individuels. Recherches sociographiques, vol. 45, n 3, pp. 427-439.

Guéraut É. (2017). Mobiliser ses capitaux d'un espace à l'autre. Le retour qualifié dans les villes moyennes. Espaces et sociétés, vol. $168-169, \mathrm{n}^{\circ} 1$, p. 51.

Guillon V., Ambrosino C. (2016). Penser la métropole à "l'âge du faire": Création numérique, éthique hacker et scène culturelle. L'Observatoire, la revue des politiques culturelles, $\mathrm{n}^{\circ} 47$.

Huriot J.-M., Bourdeau-Lepage L. (2009). Économie des villes contemporaines. Paris, Economica.
Krugman P. (1991). Increasing Returns and Economic Geography. Journal of Political Economy, vol. 99, n 3, pp. 483-499.

Lecat G. (2004). En quoi le cadre de vie rural contribue-t-il à expliquer les prix fonciers dans les espaces périurbains? Revue d'économie régionale \& urbaine, février, $\mathrm{n}^{\circ} 1$, p. 29.

Levy R., Sibertin-Blanc R., Jegou L. (2013). La production scientifique universitaire dans les villes françaises petites et moyennes (19802009), M@ppemonde, n 110.

Mainet H. (2011). Les petites villes françaises en quête d'identité. Ambiguïté du positionnement ou image tactiquement combinée ? Mots, Les langages du politique, $\mathrm{n}^{\circ} 97$.

Malmberg A. (1997). Industrial geography: location and learning. Progress in Human Geography, vol. 21, n 4 , pp. 573-582.

Mayneris F. (2017). Effets des infrastructures de transport sur le niveau et la localisation des activités économiques. Université catholique de Louvain, Discussion, 2017, n 23.

McGranahan D., Wojan T., Lambert D. (2011). The rural growth trifecta: outdoor amenities, creative class and entrepreneurial context. Journal of Economic Geography, vol. 11, $\mathrm{n}^{\circ} 3$, pp. 529-557.

Ménage P. (2011). Mise en réseau d'acteurs et compétitivité territoriale. Thèse de doctorat, Université François Rabelais, Tours.

Miles M., Huberman A. M. (1991). Analyse des données qualitatives. Bruxelles, De Boeck.

Miles M. B., Huberman A. M., Saldaña J. (2013). Qualitative data analysis: a methods sourcebook, London, Sage.

Mora O., Riba G., Hubert B. (2012). Les nouvelles ruralités? Territoires 2040, $\mathrm{n}^{\circ} 2$, pp. 93-99.

Nadou F. (2010). La notion de « villes intermédiaire », une approche différenciée du rôle des villes moyennes : entre structuration territoriale et spécificités socio-économiques. Colloque Villes petites et moyennes, un regard renouvelé, Tours.

Naldi L., Nilsson P., Westlund H., Wixe S. (2015). What is smart rural development? Journal of Rural Studies, vol. 40, pp. 90-101.

Perrier-Cornet P., Hervieu B. (2002). Campagnes Françaises multifonctionnelles, 
RECHERCHE

Geoffroy LABROUCHE, Rachel LEVY

Les enjeux de la gestion de l'espace rural. Économie \& Humanisme, $\mathrm{n}^{\circ} 362$, pp. 18-23.

Pezzini M. (2001). Rural policy lessons from OECD countries. International Régional Science Review, vol. 24, n 1, pp. 134-145.

Porter M. (1998). Clusters and new economics of competition. Harvard Business Review, vol. 76, n 6, pp. 77-90.

Reynaud J.-P., Bouvet S., (2016). Les métropoles concentrent les arrivées d'emplois et d'actifs qualifiés. INSEE analyses AuvergneRhône-Alpes, ${ }^{\circ} 28$.

Santamaria F. (2000). La notion de «ville moyenne» en France, en Espagne et au Royaume-Uni. The notion of «mediumsized town » in France, Spain and the United Kingdom. Annales de Géographie, vol. 109, $\mathrm{n}^{\circ}$ 613, pp. 227-239.

Satterthwaite D., Tacoli C. (2003). The urban part of rural development : the role of small and intermediate urban centres in rural and regional development and poverty reduction. International Institute for Environment and Devlopment.

Shah S. K., Corley K. G. (2006). Building Better Theory by Bridging the QuantitativeQualitative Divide. Journal of Management Studies, vol. 43, n 8 , pp. 1821-1835.
Tallec J. (2012). Les trajectoires économiques et les formes sociales de l'innovation dans les villes moyennes : les cas d'Albi (MidiPyrénées), d'Alès (Languedoc-Roussillon), de Fougères et de Quimper (Bretagne). Revue d'économie régionale \& urbaine, avril, ${ }^{\circ} 2$, p. 192.

Thietart R-A. (2014). Méthodes de recherche en management. Paris, Dunod.

Torre A., Pham H. V. (2015). Des usines, des champs et des villes: maillage territorial et polarisation régionale. In Rastoin J.-L., Bouquery J.-M. (dir.), Les industries agroalimentaires en France, Paris, La Documentation Française, 253 p.

Triboulet P., Pérès S. (2015). La répartition spatiale des industries agroalimentaires dans le secteur coopératif français. Économie rurale, ${ }^{\circ} 346$, pp. 49-69.

Yin R. K. (2014). Case Study Research: Design and Methods. $5^{\mathrm{e}}$ édition, Newbury Park, Sage.

Zuliani J.-M. (2004). L'organisation des services aux entreprises dans les villes moyennes du Grand Sud-Ouest français, entre logiques gravitaires et maillages interurbains. Géocarrefour, vol. 79, $\mathrm{n}^{\circ} 2$, pp. 183-192. 


\section{ANNEXE \\ Guide d'entretien : réseaux d'acteurs et territoire dans les projets innovants de l'agroalimentaire en ville moyenne \\ Aborder les conditions de confidentialité et d'enregistrement de l'entretien \\ L'étude sera adressée à l'issue du travail}

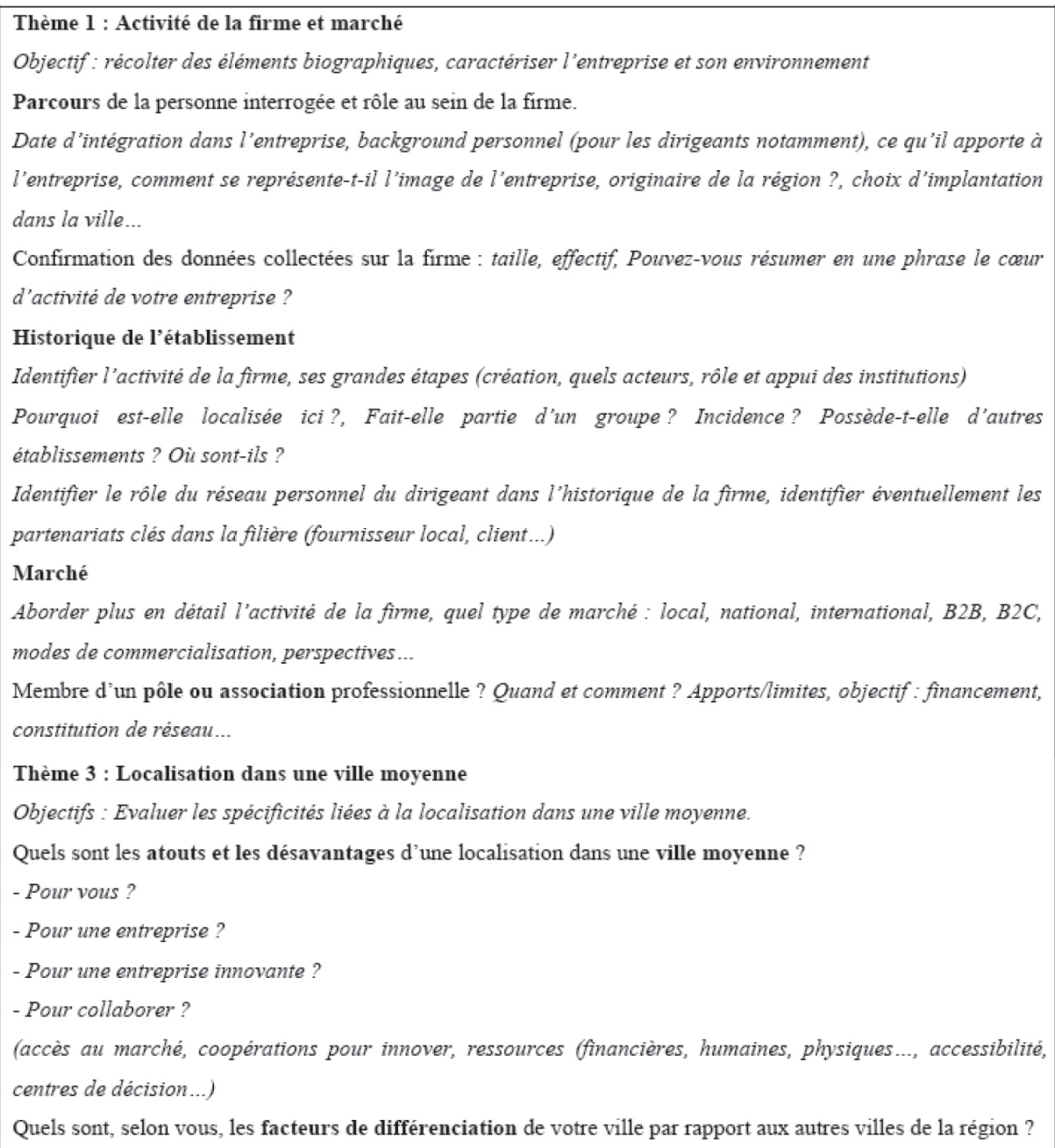

